\title{
Soluções Computacionais para Avaliação da Aprendizagem por Mapas Conceituais: um Mapeamento Sistemático
}

\author{
Silvio L. B. Boss ${ }^{1,2}$, Ecivaldo S. Matos ${ }^{2}$, Aline M. S. Andrade ${ }^{2}$ \\ ${ }^{1}$ Departamento Acadêmico de Informática \\ Universidade Tecnológica Federal do Paraná (UTFPR) \\ 85.503-390 - Pato Branco - PR - Brasil \\ ${ }^{2}$ Departamento de Ciência da Computação \\ Universidade Federal da Bahia (UFBA) \\ 40.170-110 - Salvador - BA - Brasil \\ silvioboss@utfpr.edu.br, \{aline, ecivaldo\}@ufba.br
}

\begin{abstract}
Conceptual maps have several pedagogical resources that make them a promising tool for teaching, learning and assessing knowledge. In this article, we present partial results of a systematic mapping of the literature whose objective was to identify studies and researches regarding computational solutions that have been used to evaluate the learning through the conceptual mapping. The results show the existence of challenges to evaluate conceptual maps that considers its construction process.
\end{abstract}

Resumo. Os mapas conceituais possuem vários recursos pedagógicos que os tornam uma ferramenta promissora para o ensino, a aprendizagem e avaliação do conhecimento. Neste artigo, apresentamos resultados parciais de um mapeamento sistemático da literatura cujo objetivo foi identificar estudos e pesquisas referentes a soluções computacionais que têm sido utilizadas para avaliação da aprendizagem por meio do mapeamento conceitual. Os resultados mostram a existência de desafios para avaliar mapas conceituais que consideram seu processo de construção.

\section{Introdução}

Avaliação da aprendizagem é uma tarefa bastante complexa principalmente quando se almeja por uma automatização do processo avaliativo, que requer, dentre outras necessidades, uma formalização das estruturas de representação do conhecimento. Além disso, é desejado que um processo avaliativo seja utilizado como uma prática da construção do conhecimento, melhorando tanto o ensino quanto a aprendizagem [Novak and Gowin 1984].

Mapas Conceituais (MCs), criado por Joseph D. Novak [Novak and Gowin 1984], são diagramas com organização hierárquica que apresentam relações entre conceitos, ou entre palavras que representam conceitos [Moreira 2010]. São amplamente utilizados, inclusive no âmbito da computação [Canas et al. 2018], para facilitar o processo da aprendizagem, sendo um importante instrumento para o ensino e avaliação do conhecimento construído. Quando utilizados para avaliação da aprendizagem apresentam a vantagem de fornecer evidências sobre a estrutura cognitiva do aprendiz [Moreira 2010].

Existem diversos desafios no uso de mapas conceituais como instrumento de aprendizagem e avaliação, devido principalmente a natureza intrínseca dos processos 
VIII Congresso Brasileiro de Informática na Educação (CBIE 2019)

Anais do XXX Simpósio Brasileiro de Informática na Educação (SBIE 2019)

cognitivos (próprios de cada sujeito). Pesquisas no campo da Informática na Educação têm investigado o problema de se avaliar a aprendizagem de alunos comparando o MC de cada aprendiz com um mapa de referência, por meio de ferramentas computacionais [Chang et al. 2005, Lukasenko and Grundspenkis 2010, Gaspar et al. 2018]. Outros trabalhos [Anohina and Grundspenkis 2007, Caldas and Favero 2009, Gurupur et al. 2015] têm mostrado, ao longo dos anos, que automatizar a avaliação da aprendizagem por meio da análise de mapas não é um processo simples. Isto se deve a determinados aspectos levados em consideração quando feita a comparação entre os mapas, como é o caso da análise estrutural, para reconhecer possíveis padrões de soluções, e da análise semântica, para o reconhecimento dos significados.

A identificação e análise das soluções que têm sido desenvolvidas para avaliação da aprendizagem por mapas conceituais são importantes para que novas estratégias possam ser propostas, inclusive computacionais. Nesse sentido, este artigo apresenta os resultados parciais de um mapeamento sistemático, cujo objetivo foi identificar quais soluções têm sido utilizadas para avaliação da aprendizagem por meio do mapeamento conceitual.

Este texto está estruturado da seguinte forma: na Seção 2 discutimos sobre o referencial teórico que trata da avaliação de mapas conceituais. Na Seção 3 são apresentados os trabalhos relacionados. Na Seção 4 é discutido o protocolo utilizado para o processo de condução do mapeamento. Na Seção 5 é apresentada a análise dos resultados alcançados com a condução do mapeamento. A Seção 6 apresenta os resultados e uma breve discussão do mapeamento. Por fim, na Seção 7 é apresentada a conclusão.

\section{Avaliação de Mapas Conceituais}

Mapas conceituais são resultantes do processo de mapeamento conceitual, alicerçado na teoria da Aprendizagem Significativa, proposta por Ausubel. A aprendizagem significativa é definida como um processo por meio do qual uma nova informação se relaciona, de maneira substantiva e não arbitrária, a um aspecto relevante da estrutura de conhecimento do indivíduo [Ausubel 2003]. Nesta perspectiva, a Teoria de Ausubel define aspectos cognitivos como a subsunção, reconciliação integrativa e diferenciação progressiva, que são denominados, neste texto, de categorias-chave.

O processo de subsunção, descrito por Ausubel em sua teoria, é chamado de princípio da assimilação. É um processo que ocorre quando um determinado conceito $a$, que é potencialmente significativo, é assimilado sob um conceito $b$ (chamado de subsunçor) mais inclusivo, já existente na estrutura cognitiva do indivíduo, produzindo um novo significado, resultado da interação entre ambos. A ocorrência deste processo iteradas vezes na estrutura cognitiva do indivíduo resulta na diferenciação progressiva. A reconciliação integrativa é a associação entre conceitos previamente existentes na estrutura cognitiva do indivíduo. Neste caso, os conceitos são reconhecidos e relacionados de modo diferente da anteriormente existente, gerando novos significados a partir da reorganização destes conceitos [Ausubel 2003].

A representação do conhecimento, por meio do mapeamento conceitual, é uma alternativa para estruturar a informação, uma vez que é possível representar a organização da estrutura cognitiva de um indivíduo sobre um determinado domínio. Consequentemente, essas estruturas de conhecimento são representações da organização de conceitos 
VIII Congresso Brasileiro de Informática na Educação (CBIE 2019)

Anais do XXX Simpósio Brasileiro de Informática na Educação (SBIE 2019)

e ideias presentes na estrutura cognitiva do indivíduo [da Silva 2015].

Como ferramenta de avaliação, os mapas conceituais podem ser usados para obter uma visualização da organização conceitual que é atribuída a um determinado conhecimento [Chang et al. 2005]. Na pesquisa de [da Silva 2015], Silva afirma que um dos maiores desafios para utilização dos mapas conceituais está no processo de avaliação, em virtude de o mapa ser elaborado baseado na percepção de aprendizagem de cada indivíduo. Ou seja, tanto os mapas conceituais elaborados por professores quanto os mapas elaborados pelos aprendizes possuem componentes idiossincráticos. Isso significa dizer que não existe um único mapa correto. Esse é um desafio frequentemente encontrado na literatura quando almejamos realizar avaliações da aprendizagem por mapas conceituais. Pois, a liberdade permitida na construção de um mapa, por meio de conceitos e relações, faz com que a mesma informação possa ser representada de formas diferentes quando definidas por diferentes indivíduos [da Rocha et al. 2004].

Neste contexto, é importante utilizar e propor soluções que possam fornecer subsídios a um processo de avaliação para apoiar professores e alunos na construção e avaliação do conhecimento.

\section{Trabalhos Relacionados}

Foram encontrados três trabalhos correlatos, ou seja, revisões bibliográficas sobre técnicas, ferramentas e tipos de tarefas adotados para analisar mapas conceituais.

Em [Strautmane 2012], o autor apresenta uma revisão de literatura sobre os tipos de tarefas mais frequentemente empregados na construção de mapas conceituais aplicados na avaliação da aprendizagem, bem como os critérios utilizados para analisá-los. Estes critérios são aqueles propostos por Novak e Gowin [Novak and Gowin 1984]. No trabalho de [Naumeca and Grundspenkis 2009], os autores apresentam um resumo e classificação dos sistemas de pontuação propostos para avaliar mapas conceituais, presentes na literatura. O foco do artigo é mapear os sistemas de pontuação utilizados para uma avaliação automatizada baseada em mapas conceituais. Por sua vez, em [Canas et al. 2003], os autores apresentam uma revisão da literatura sobre as áreas de aplicação (i.e., educação, comercial e governamental), e as técnicas e as ferramentas utilizadas para apoiar a aprendizagem por mapas conceituais. No entanto, esse trabalho não tem foco nos aspectos computacionais, tampouco na avaliação da aprendizagem.

Apesar desses trabalhos apresentarem revisões de literatura sobre técnicas para analisar mapas conceituais estes não seguiram um protocolo de mapeamento sistemático de literatura como o nosso. Nosso mapeamento também não é restrito a contextos específicos, como nos dois primeiros trabalhos acima que tratam especificamente ou de tipos de tarefas ou sistemas de pontuação. Neste sentido, apresentamos como principal contribuição deste estudo em relação aos citados anteriormente a busca de trabalhos que abordam soluções que analisam mapas conceituais tanto de forma qualitativa quanto quantitativa.

\section{Metodologia do Mapeamento}

Este mapeamento sistemático de literatura tem por objetivo:

(OE1) Identificar soluções computacionais utilizadas para avaliação da aprendizagem por meio do mapeamento conceitual. 
VIII Congresso Brasileiro de Informática na Educação (CBIE 2019)

Anais do XXX Simpósio Brasileiro de Informática na Educação (SBIE 2019)

(OE2) Identificar lacunas de pesquisa que possam existir nos estudos encontrados.

O primeiro objetivo trata do levantamento de resultados sobre aprendizagem em geral e também sobre aprendizagem significativa através da identificação de trabalhos que utilizam as categorias-chave (OE1). O segundo objetivo trata da identificação de lacunas nas soluções que avaliam a aprendizagem, para a posteriori, propor novas soluções (OE2). Para isso, nos baseamos nas diretrizes propostas por Kitchenham e Charters [Kitchenham and Charters 2007]. Em conformidade com essas diretrizes são apresentados a seguir os principais pontos metodológicos deste mapeamento, a saber: questão de pesquisa, estratégia de busca e seleção, critérios de inclusão e exclusão e seleção e classificação.

\subsection{Questão de Pesquisa}

Seguimos a seguinte estrutura para definir a questão de pesquisa, conforme descrito em [Petticrew and Roberts 2006]:

- População: conjunto de artigos em bases específicas sobre avaliação da aprendizagem por mapas conceituais.

- Intervenção: soluções, práticas utilizadas para avaliar a aprendizagem por intermédio de mapas conceituais.

- Comparação: não se aplica.

- Resultado: uma abordagem utilizada para avaliar a aprendizagem por meio de mapas conceituais e lacunas de pesquisa na área.

- Contexto: avaliação da aprendizagem por mapas.

Questão de Pesquisa: Quais soluções computacionais foram desenvolvidas para avaliação da aprendizagem por meio de mapas conceituais?

- Rationale: a questão de pesquisa foi definida com o propósito de identificar estudos que apresentem soluções computacionais para avaliação da aprendizagem por meio de mapas conceituais.

- Hipótese: existem estudos que apresentem soluções computacionais para avaliação da aprendizagem por meio de mapas conceituais.

- Hipótese negativa: não há estudos que apresentem soluções computacionais para avaliação da aprendizagem por meio de mapas conceituais.

\subsection{Estratégia de Busca e Seleção}

A estratégia de busca e a seleção dos artigos foram definidos a partir:

- Bases de dados: Compendex Engineering Index, Web of Science, IEEE Xplore Digital Library, ACM Digital Library e Portal de Publicações da CEIE;

- Termos da busca: evaluation, assessment, concept maps, concept mapping.

Nossa pesquisa incluiu trabalhos escritos em inglês e em português. Neste sentido, o Quadro 4.1 apresenta nossa string base em inglês utilizada para buscar os trabalhos nas bases de dados: 
VIII Congresso Brasileiro de Informática na Educação (CBIE 2019)

Anais do XXX Simpósio Brasileiro de Informática na Educação (SBIE 2019)

("concept maps"or "concept mapping") and (assessment or evaluation)

Quadro 4.1: string base em inglês.

\subsection{Critérios de Inclusão e Exclusão}

Estabelecemos um conjunto de critérios de inclusão e exclusão para avaliar cada trabalho, os quais foram especificados com base na questão de pesquisa para garantir que trabalhos relacionados ao contexto deste mapeamento fossem selecionados como estudos primários.

- Critérios de Inclusão:

- CI1. Estudo, cujo objeto de pesquisa esteja relacionado à avaliação da aprendizagem por meio de mapas conceituais;

- CI2. Estudo que apresente solução(ões) computacional(ais) utilizada(as) para avaliação da aprendizagem por meio do mapeamento conceitual.

- Critérios de Exclusão:

- CE1. Artigo duplicado.

- CE2. Artigo considerado de literatura cinza ${ }^{1}$.

- CE3. Artigo com menos de cinco páginas (short paper).

- CE4. Estudo secundário (revisão e mapeamento de literatura).

- CE5. Artigo indisponível (de acesso restrito ou não encontrados).

- CE6. Artigo com insuficiência de conteúdo para os objetivos deste mapeamento.

- CE7. Artigo escrito em idioma diferente do inglês ou português.

\subsection{Seleção e Classificação}

As buscas nas bases de dados retornaram um total de 890 trabalhos (considerando os artigos duplicados). A Figura 1 apresenta a quantidade de trabalhos encontrados em cada base, apresentando o percentual em relação ao total. Após excluir os artigos duplicados, ficaram 735 trabalhos. Dando-se início as subetapas da execução do mapeamento.

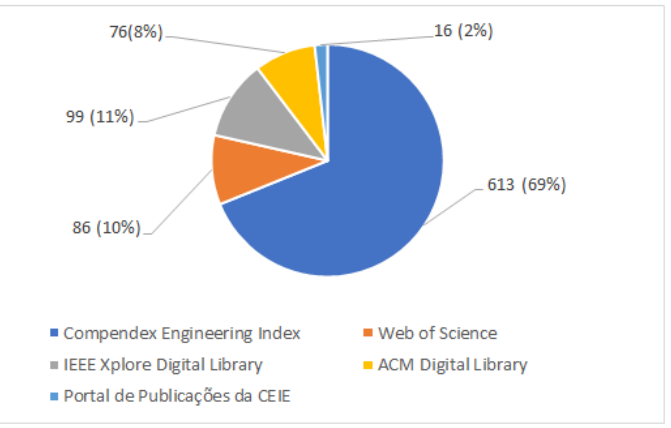

Figura 1. Quantidade de artigos encontrados em cada base.

Fonte: Elaborado pelo autor.

Dividimos a seleção e classificação em quatro etapas. Na etapa 1 os artigos foram buscados nas bases de dados e em anais de conferência. Na etapa 2, foram aplicados os critérios de inclusão e exclusão. Como critério de seleção dos artigos, foram utilizados

\footnotetext{
${ }^{1}$ Foram considerados de literatura cinza: relatórios (técnicos, preliminar, avançado), artigos que não têm foco científico em seu conteúdo, como os editoriais, prefácios, discussões, resenhas e resumo de tutoriais.
} 
VIII Congresso Brasileiro de Informática na Educação (CBIE 2019)

Anais do XXX Simpósio Brasileiro de Informática na Educação (SBIE 2019)

os metadados título e resumo de cada artigo. Para isso, utilizou-se o Mendeley ${ }^{2}$ como ferramenta de suporte. Estudos identificados como relevantes, foram selecionados para a etapa 3.

Na etapa 3 realizou-se a leitura das seções de introdução e conclusão de todos os trabalhos aceitos na fase anterior. Os artigos, cuja leitura da introdução e da conclusão não foram suficientes para o julgamento de inclusão ao mapeamento, foram lidos na íntegra por meio das técnicas de leitura dinâmica, skimming e scanning, por não exigirem leitura precisa e detalhada do texto por completo. Com o objetivo de estabelecer uma classificação entre os artigos selecionados, considerando sua relevância para o alcance dos objetivos do mapeamento, foram aplicados os seguintes critérios de qualidade: (i) a estrutura é adequada e de fácil compreensão?; (ii) existe uma apresentação clara dos objetivos da pesquisa?; (iii) o problema de pesquisa é claramente definido?; (iv) a metodologia de pesquisa é claramente descrita?; (v) existe uma apresentação clara dos resultados?; (vi) as técnicas e métodos para avaliar a aprendizagem por mapas conceituais são descritos?; (vii) o processo para avaliar a aprendizagem por mapas conceituais é descrito?

$\mathrm{Na}$ etapa 4 obtivemos os artigos para leitura, análise e discussão. Nesta etapa, os trabalhos foram lidos na íntegra para obtenção dos dados que atendessem aos objetivos do mapeamento sistemático, considerando os critérios de qualidades definidos pelos pesquisadores. A Figura 2 apresenta o número de artigos encontrados nas buscas iniciais, depois selecionados em cada subetapa da execução deste mapeamento.

\begin{tabular}{|c|c|c|c|c|}
\hline $\begin{array}{l}\text { Estudos } \\
\text { retornados pela } \\
\text { string de busca } \\
\quad(\mathrm{n}=890)\end{array}$ & $\begin{array}{c}\text { Após eliminar } \\
\text { estudos } \\
\text { duplicados } \\
(\mathrm{n}=735)\end{array}$ & $\begin{array}{c}\text { Após leitura } \\
\text { do título e } \\
\text { abstract } \\
(\mathrm{n}=103)\end{array}$ & $\begin{array}{l}\text { Após leitura } \\
\text { da introdução } \\
\text { e conclusão } \\
(\mathrm{n}=71)\end{array}$ & $\begin{array}{l}\text { Artigos } \\
\text { primários } \\
\text { obtidos } \\
(\mathrm{n}=46)\end{array}$ \\
\hline
\end{tabular}

Figura 2. Quantidade de artigos selecionados.

Fonte: Elaborado pelo autor.

\section{Análise dos Resultados}

Esta seção resume os principais resultados encontrados neste estudo e discute a sua relevância para a área de Informática na Educação. Para responder nossa questão de pesquisa, primeiramente classificamos os trabalhos encontrados da seguinte forma: especificação formal, comparação entre mapas, sistema de pontuação e uso de semântica. Apresentamos a tabulação dos dados encontrados nos artigos primários selecionados. A Seção 6 discute quais as soluções encontradas neste mapeamento.

- Especificação formal. Procuramos saber se o artigo faz algum mapeamento do mapa conceitual para alguma estrutura formal;

- Comparação entre mapas. Neste caso, estamos interessados em saber se a avaliação é feita por meio da comparação entre mapas, por exemplo, entre um mapa de referência e um mapa do aprendiz;

- Sistema de pontuação. Neste item, procuramos averiguar se na avaliação é utilizado algum sistema de pontuação;

- Uso de semântica. Aqui, pesquisamos se os trabalhos utilizam alguma forma de verificar o uso de sinônimos nos conceitos ou nas palavras de ligação.

\footnotetext{
${ }^{2} \mathrm{https}: / / \mathrm{www} \cdot \mathrm{mendeley.com/}$
} 
VIII Congresso Brasileiro de Informática na Educação (CBIE 2019)

Anais do XXX Simpósio Brasileiro de Informática na Educação (SBIE 2019)

A Figura 3 apresenta os dados tabulados referente aos 46 artigos selecionados neste mapeamento. A partir do uso das características definidas para análise das abordagens, como descrito anteriormente, é possível uma compreensão mais aprofundada das pesquisas na área da avaliação da aprendizagem por mapas conceituais.

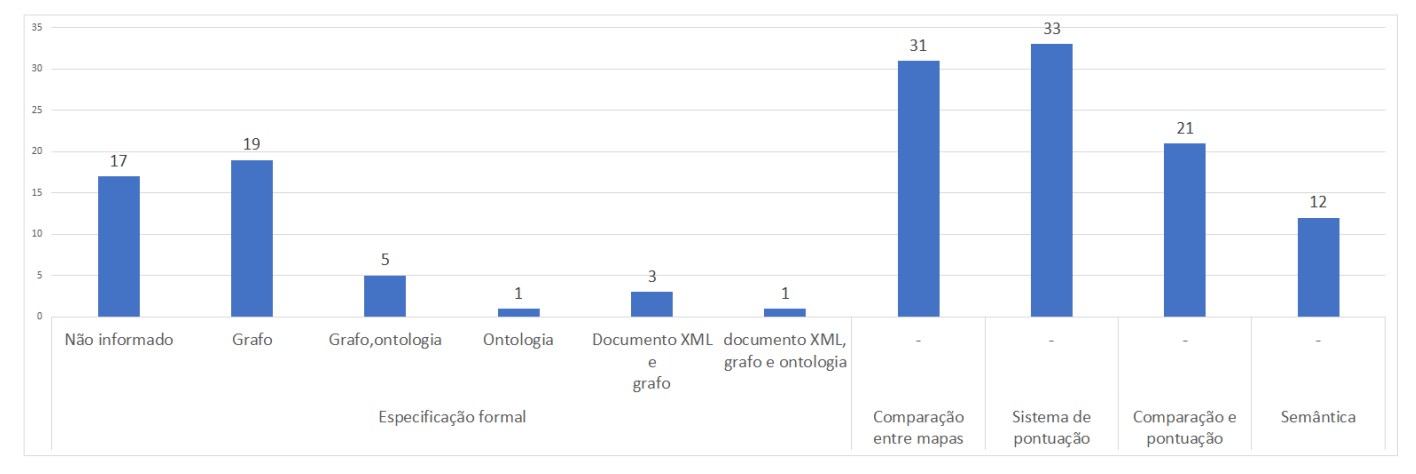

Figura 3. Tabulação dos dados referente aos trabalhos selecionados.

Fonte: Elaborado pelo autor.

$\mathrm{Na}$ figura acima apresentamos os tipos mais comuns para especificar formalmente mapas conceituais. Dos 46 trabalhos, em relação à especificação formal não existe interseção entre cada tipo tabulado, ou seja, um mesmo artigo só aparece em somente um tipo. Em relação aos outros dados, somente o tipo semântica tem interseção com outros dados tabulados. Na próxima seção detalhamos os aspectos utilizados para classificação dos artigo em cada tipo.

\section{Resultados e Discussão}

Este mapeamento sistemático teve por objetivo investigar soluções para avaliação da aprendizagem por mapas conceituais como forma de responder a questão de pesquisa definida na Seção 4.1. Neste sentido, este trabalho identificou um conjunto de soluções que são relevantes para realizar uma avaliação qualitativa (análise estrutural e semântica) e quantitativa (sistema de pontuação). Analisando os trabalhos deste mapeamento, foi possível encontrar algumas informações que envolveram a avaliação da aprendizagem por mapas conceituais, tais como: (i) Análise estrutural - podemos citar alguns trabalhos como, por exemplo, os trabalhos de Anohina e Grundspenkis [Anohina and Grundspenkis 2007, Grundspenkis 2008, Grundspenkis 2009], que desenvolveram um sistema inteligente de avaliação de conhecimento, denominado de IKAS $^{3}$, estes definem padrões de comparação para determinar a correção de cada proposição no mapa do aprendiz em relação ao mapa de referência. Já os trabalhos de Jain e Gurupur [Jain et al. 2014, Gurupur et al. 2015] utilizam a técnica de parser XML para realizar avaliação de mapas conceituais; (ii) Análise semântica - foram encontrados trabalhos que fazem avaliações levando em consideração não apenas os aspectos estruturais como também os semânticos. Podemos citar as pesquisas de [da Rocha et al. 2004, da Rocha et al. 2005, Limongelli et al. 2017] como exemplos de trabalhos que utilizam ontologias de domínio para auxiliar na automatização da avaliação da aprendizagem mediada por mapas conceituais e que consideram a semântica das relações. O trabalho de

\footnotetext{
${ }^{3}$ Intelligent Knowledge Assessment System.
} 
VIII Congresso Brasileiro de Informática na Educação (CBIE 2019)

Anais do XXX Simpósio Brasileiro de Informática na Educação (SBIE 2019)

[Caldas and Favero 2009] utiliza um dicionário de sinônimos para representar os conceitos e as palavras de ligação; (iii) Sistema de pontuação - a estratégia mais usada em pesquisas para pontuar componentes de um mapa conceitual é o método de pontuação desenvolvido por Novak e Gowin [Novak and Gowin 1984]. Esse método também é usado como base para outros sistemas avaliativos quantitativos, como é o caso, por exemplo, dos trabalhos de [Sacre et al. 2013, Jain et al. 2014, Watson et al. 2016]. Alguns trabalhos utilizam medidas de similaridade entre mapas conceituais para calcular a similaridade entre os mapas como é o caso dos trabalhos de [Chang et al. 2005, Chen et al. 2001] que utilizam um método teórico, denominado de medida $\mathrm{C}$, para quantificar a similaridade entre dois mapas conceituais. O trabalho de [Iqbal et al. 2018] utiliza uma técnica que combina duas medidas diferentes: índice de proximidade [Goldsmith et al. 1991] e índice de similaridade [Chang et al. 2005]. Também foi encontrado um trabalho [Al Duhayyim and Newbury 2018] sobre um sistema, denominado $\mathrm{CaFAE}^{4}$, que utiliza lógica fuzzy para avaliar e mostrar o nível de conhecimento do aluno para cada conceito do mapa em um domínio de conhecimento.

Este mapeamento ainda encontrou trabalhos que fazem uso das categorias-chave de Ausubel para realizar avaliação da aprendizagem significativa. Este é o caso de [Watson et al. 2016] sobre uma análise qualitativa utilizando métodos de pontuação que analisa: (i) grau de subsunção, avaliado pelos níveis hierárquicos do mapa, denominado pelos autores de profundidade do conhecimento; (ii) diferenciação progressiva, avaliada pelas ramificações, denominado de amplitude do conhecimento; (iii) reconciliação integrativa, avaliada pelas ligações cruzadas no mapa. Outros trabalhos que fazem avaliação da aprendizagem significativa usando mapas conceituais por meio das categorias-chaves de Ausubel são: [da Rocha et al. 2004, Sacre et al. 2013, da Silva and Omar 2017].

\section{Conclusão}

Este artigo apresentou resultados parciais de um mapeamento sistemático da literatura, com o objetivo de identificar estudos e pesquisas referente a soluções que têm sido utilizadas para avaliação da aprendizagem por meio do mapeamento conceitual.

O mapeamento foi realizado por meio de um protocolo de revisão que especificou os métodos utilizados durante a realização do trabalho. Os critérios definidos no protocolo foram necessários e suficientes para obter os estudos primários necessários para realizar a pesquisa. Por meio deste mapeamento foi possível obter resposta à questão de pesquisa levantada neste trabalho.

Esta pesquisa identificou soluções que podem ser usadas para avaliar tanto aspectos estruturais quanto semânticos na avaliação de mapas conceituais como também as categorias-chaves de Ausubel. No entanto, este mapeamento não encontrou soluções computacionais que avaliassem a aprendizagem dentro de um processo de construção de mapas conceituais considerando a idiossincrasia de mapas e que coadunassem uma análise estrutural e semântica, as categorias-chaves de Ausubel e um sistema de pontuação.

\section{Referências}

Al Duhayyim, M. and Newbury, P. (2018). Concept-based and fuzzy adaptive e-learning. In Proceedings of the 2018 The 3rd International Conference on Information and Edu-

\footnotetext{
${ }^{4}$ Concept and Fuzzy Adaptive E-learning.
} 
VIII Congresso Brasileiro de Informática na Educação (CBIE 2019)

Anais do XXX Simpósio Brasileiro de Informática na Educação (SBIE 2019)

cation Innovations, ICIEI 2018, pages 49 - 56, New York, NY, USA. ACM.

Anohina, A. and Grundspenkis, J. (2007). A concept map based intelligent system for adaptive knowledge assessment. In Proceedings of the Conference on Databases and Information Systems, pages 263-276, Amsterdam, The Netherlands. IOS Press.

Ausubel, D. (2003). Aquisição e retenção de conhecimentos: Uma perspectiva cognitiva. Platano Edições Técnicas.

Caldas, V. M. and Favero, E. L. (2009). Uma ferramenta de avaliação automática para mapas conceituais como auxílio ao ensino em ambientes de educação a distância. In Anais do simpósio brasileiro de informática na educação - SBIE, Florianópolis. XX Simpósio brasileiro de informática na educação, UFSC.

Canas, A. J., Carff, R., and Lott, J. (2018). eCMap: An embeddable web-based concept map editor. In Proceedings of the Eighth International Conference on Concept Mapping, Medellín, Colombia.

Canas, A. J., Coffey, J. W., Carnot, M. J., Feltovich, P., Hoffman, R. R., Feltovich, J., and Novak, J. D. (2003). A summary of literature pertaining to the use of concept mapping techniques and technologies for education and performance support. Technical report, The Institute for Human and Machine Cognition - Pensacola, Florida.

Chang, K., Sung, Y., Chang, R., and Lin, S. (2005). A new assessment for computer-based concept mapping. Educational Technology \& Society, 8:138-148.

Chen, S. W., Lin, S. C., and Chang, K. E. (2001). Attributed concept maps: fuzzy integration and fuzzy matching. IEEE Transactions on Systems, Man, and Cybernetics, Part B (Cybernetics), 31(5):842-852.

da Rocha, F. E. L., da Costa Junior, J. V., and Favero, E. L. (2005). Como usar ontologias na avaliação da aprendizagem significativa mediada por mapas conceituais. Revista Brasileira de Informática na Educação, 13(2):53-64.

da Rocha, F. E. L., Vieira, R. V., da Costa Jr, J. V., and Favero, E. L. (2004). Especificação de um algoritmo genético para auxiliar na avaliação da aprendizagem significativa com mapas conceituais. In Anais do simpósio brasileiro de informática na educação, pages 139-148, Manaus. XV Simpósio brasileiro de informática na educação, UFAM.

da Silva, E. C. (2015). Mapas conceituais: propostas de aprendizagem e avaliação. Administração: Ensino e Pesquisa, 16(4).

da Silva, V. and Omar, N. (2017). Model of measurement of meaningful learning in distance learning environments. In 12th Iberian Conference on Information Systems and Technologies (CISTI), pages 1-6.

Gaspar, W., Aguiar, C. Z., Gava, T. B. S., and Cury, D. (2018). Uma arquitetura tecnológica para apoiar o professor na utilização de mapas conceituais em sala de aula. In Anais do simpósio brasileiro de informática na educação, pages 158-167, Fortaleza, CE. Universidade Federal do Ceará - UFC.

Goldsmith, T. E., Johnson, P. J., and Acton, W. H. (1991). Assessing structural knowledge. Journal of Educational Psychology, 83:88-96. 
VIII Congresso Brasileiro de Informática na Educação (CBIE 2019)

Anais do XXX Simpósio Brasileiro de Informática na Educação (SBIE 2019)

Grundspenkis, J. (2008). Development of concept map based adaptive knowledge assessment system. In Multi Conference on Computer Science and Information Systems; Proceedings of e-Learning, volume 1, pages 395-402.

Grundspenkis, J. (2009). Concept maps as knowledge assessment tool: Results of practical use of intellligent knowledge assessment system. In Proceedings of the International Conference Cognition and Exploratory Learning in Digital Age, pages 258-266.

Gurupur, V. P., Jain, G. P., and Rudraraju, R. (2015). Evaluating student learning using concept maps and markov chains. Expert Systems with Applications, 42(7):3306-3314.

Iqbal, R., Murad, M. A. A., Sliman, L., and da Silva, C. P. (2018). A mathematical evaluation for measuring correctness of domain ontologies using concept maps. Measurement, $118: 73-82$.

Jain, G. P., Gurupur, V. P., Schroeder, J. L., and Faulkenberry, E. D. (2014). Artificial intelligence-based student learning evaluation: A concept map-based approach for analyzing a student's understanding of a topic. IEEE Transactions on Learning Technologies, 7(3):267-279.

Kitchenham, B. A. and Charters, S. (2007). Guidelines for performing systematic literature reviews in software engineering. Technical report, School of Computer Science and Mathematics, Keele University, Keele, UK.

Limongelli, C., Sciarrone, F., Lombardi, M., Marani, A., and Temperini, M. (2017). A framework for comparing concept maps. In 16th International Conference on Information Technology Based Higher Education and Training (ITHET), pages 1-6.

Lukasenko, R. and Grundspenkis, J. (2010). Adaptation of intelligent knowledge assessment system based on learner's model. In 16th International Conference on Information and Software Technologies.

Moreira, M. A. (2010). Mapas conceituais e a aprendizagem significativa. Centauro Editora, São Paulo, SP.

Naumeca, A. A. and Grundspenkis, J. (2009). Scoring concept maps: An overview. In Proceedings of the International Conference on Computer Systems and Technologies and Workshop for PhD Students in Computing, pages 78:1-78:6, New York, NY, USA.

Novak, J. D. and Gowin, D. B. (1984). Learning How to Learn. Cambridge University Press.

Petticrew, M. and Roberts, H. (2006). Systematic Reviews in the Social Sciences: A Practical Guide. Blackwell Publishing, Oxford.

Sacre, M. B., Gerchak, J., Lyons, M. R., Shuman, L. J., and Wolf, H. (2013). Scoring concept maps: An integrated rubric for assessing engineering education. Journal of Engineering Education, 93(2):105-115.

Strautmane, M. (2012). Concept map-based knowledge assessment tasks and their scoring criteria: an overview. In Proceedings of the Fifth International Conference on Concept Mapping, Valletta, Malta.

Watson, M. K., Pelkey, J., Noyes, C. R., and Rodgers, M. O. (2016). Assessing conceptual knowledge using three concept map scoring methods. Journal of Engineering Education, 105(1):118-146. 